\title{
Variational principles for water waves from the viewpoint of a time-dependent moving mesh
}

\author{
Thomas J. Bridges \& Neil M. Donaldson \\ Department of Mathematics, University of Surrey, Guildford, GU2 7XH, UK
}

May 21, 2010

\begin{abstract}
The time-dependent motion of water waves with a parametrically-defined free surface is mapped to a fixed time-independent rectangle by an arbitrary transformation. The emphasis is on the general properties of transformations. Special cases are algebraic transformations based on transfinite interpolation, conformal mappings, and transformations generated by nonlinear elliptic PDEs. The aim is to study the effect of transformation on variational principles for water waves such as Luke's Lagrangian formulation, Zakharov's Hamiltonian formulation, and the Benjamin-Olver Hamiltonian formulation. Several novel features are exposed using this approach: a conservation law for the Jacobian, an explicit form for surface re-parameterization, inner versus outer variations and their role in the generation of hidden conservation laws of the Laplacian, and some of the differential geometry of water waves becomes explicit. The paper is restricted to the case of planar motion, with a preliminary discussion of the extension to three-dimensional water waves.
\end{abstract}

\section{Introduction}

In the theory of water waves, the fluid domain shape is changing with time. Therefore it is appealing to transform the moving domain to a fixed domain. This approach, first for steady waves and later for unsteady waves, has been widely used in the study of water waves. However, in all cases the transformation is either algebraic (explicit) or a conformal mapping. In this paper we look at the equations governing the motion of water waves from the viewpoint of an arbitrary time-dependent transformation of the form

$$
(\mu, \nu, \tau) \mapsto\left\{\begin{array}{l}
x(\mu, \nu, \tau) \\
y(\mu, \nu, \tau) \\
t(\tau)
\end{array}\right.
$$

where $(\mu, \nu)$ are coordinates for a fixed time-independent rectangle

$$
D:=\{(\mu, \nu): 0 \leq \mu \leq L \text { and }-h \leq \nu \leq 0\},
$$

with $h, L$ given positive parameters. A more general transformation of time, $(\mu, \nu, \tau) \mapsto t(\mu, \nu, \tau)$, can be used but there does not appear to be any advantage to this generalization. A schematic of this transformation is shown in Figure 1. The transformation maps the time-dependent region $V(t)$ in the $(x, y)$ plane to the fixed region $D$ in the $(\mu, \nu)$ plane. Throughout the paper the mapping (1.1) will be a general transformation, and comments on specific choices of mapping are in $\S 9$.

The mapping (1.1) can be any smooth function satisfying the non-degeneracy conditions

$$
t^{\prime}(\tau) \neq 0 \quad \text { and } \quad J \neq 0
$$




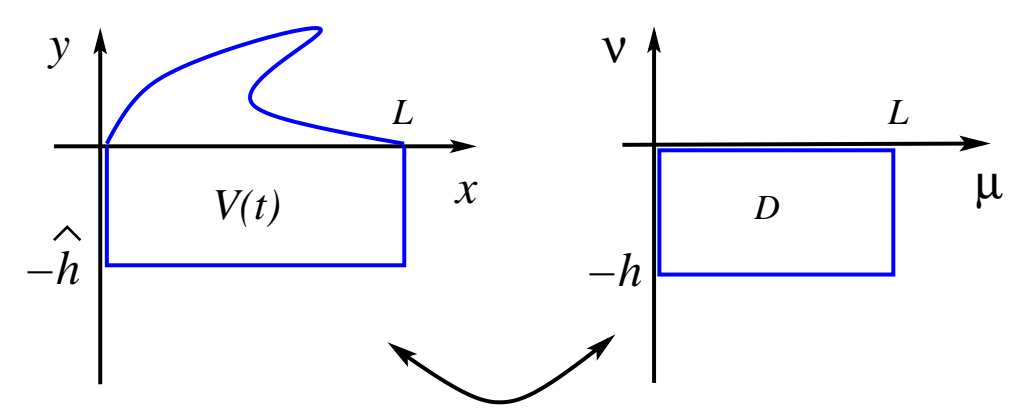

Figure 1: Schematic of the mapping of the time-dependent fluid region into a fixed rectangle.

where

$$
J:=\operatorname{det}\left[\begin{array}{ll}
x_{\mu} & x_{\nu} \\
y_{\mu} & y_{\nu}
\end{array}\right]
$$

Almost all work to date on time-dependent mappings is based on conformal maps. The first published paper on a time-dependent conformal map for water waves appears to be BYATT-SMITH [9] who derives an integral equation for the mapping, and then uses it to give a new derivation of Boussinesq-type shallow water models. Shortly thereafter, WHITNEY [36] independently proposed a numerical method for computing a time-dependent conformal map, and presented results for spatially-periodic standing waves in deep water. The formulation involved integration of an integro-differential equation with the integral part based on a Hilbert transform. The Phd thesis of GRANT [22] appeared a year later, in which a time-dependent conformal mapping was used in combination with a perturbation method to compute high-order standing waves. The first paper in the Russian literature is the work of Voinov \& Voinov [35] which appeared in 1975. (We have not seen [35] but it is mentioned by Shamin [28].)

In 1978 LongueT-Higgins [23] proposed a time-dependent conformal mapping where the independent variables are the velocity potential $(\phi)$ and stream function $(\psi)$. For steady flow the stream function is a constant at the surface, but for unsteady flow it is not. Therefore the image of the mapping in [23] is a region with a time-dependent edge, and the image of the free surface is a curve in the $(\phi, \psi)$ plane. The advantage however, is that the linearization about a Stokes wave results in a boundary-value problem in a fixed rectangular domain, and so for a stability analysis it is a very effective approach.

The next significant development is the seminal paper of DYACHENKO ET AL. [16] which had three major ideas: the introduction of a variational principle for a time-dependent conformal map, the use of the Hilbert transform to relate conjugate harmonic functions, and the introduction of a fast numerical algorithm for a spatially-periodic time-dependent conformal mapping. That theory was initially for infinite depth, but has since been extended to finite depth (cf. DyACHENko ET AL. [17] and ChOI \& CAMAssa [13]). A review article following up on this approach with extensive references has recently been published (cf. Shamin [28]).

However conformal mappings do have problems. Firstly, when the domain is a quadrilateral, the aspect ratio (conformal modulus) of the rectangle $D$ has to be computed as part of the problem, and when the mapping is time-dependent the conformal modulus is time dependent. The paper of SEIDL \& KLOSE [27] has a clear introduction to the conformal modulus problem. Secondly, conformal maps do not extend to three-dimensional water waves. Thirdly, Clammond \& Grue [14] point out that although time-dependent conformal maps are efficient, the conformality results in rough grid spacing near crests, and fine grid spacing near troughs which is the opposite of what is required. In the grid generation literature conformal maps have been largely dispensed with (cf. [33, 18]).

What other possibilities for mappings are there? There has been a considerable amount of work on this problem in the grid generation literature for both steady transformations and moving 
grids (e.g. $[10,11,18,32,33])$. Specific examples of this transformation will be discussed - such as explicit mappings and transformations generated by elliptic PDEs - but initially the general properties of variational formulations of the water wave equations in curvilinear coordinates are discussed.

The fluid occupies a simply-connected subset of $\mathbb{R}^{2}$, with coordinates $(x, y)$. In $\mathbb{R}^{2}$ the set $\Gamma(t)$, defined by

$$
\Gamma(t)=\left\{(x, y) \in \mathbb{R}^{2}: x=X(\mu, t), \quad y=Y(\mu, t), \quad 0 \leq \mu \leq L\right\},
$$

is a parametric representation of a curve in the plane for each value of $t$, and this curve represents the free surface. It is assumed throughout the paper that the surface is non-degenerate:

$$
\ell(t) \neq 0 \text { for all } t, \text { where } \ell(t)=\sqrt{X_{\mu}^{2}+Y_{\mu}^{2}}
$$

Any reasonably smooth domain shape is admissible, but for definiteness the region is taken to be rectangular except for the surface. The fluid is bounded by the surface $\Gamma(t)$, and the lines $x=0$, $x=L$ and $y=-\widehat{h}$ as shown schematically in Figure 2. In general $\widehat{h} \neq h$. The time-dependent

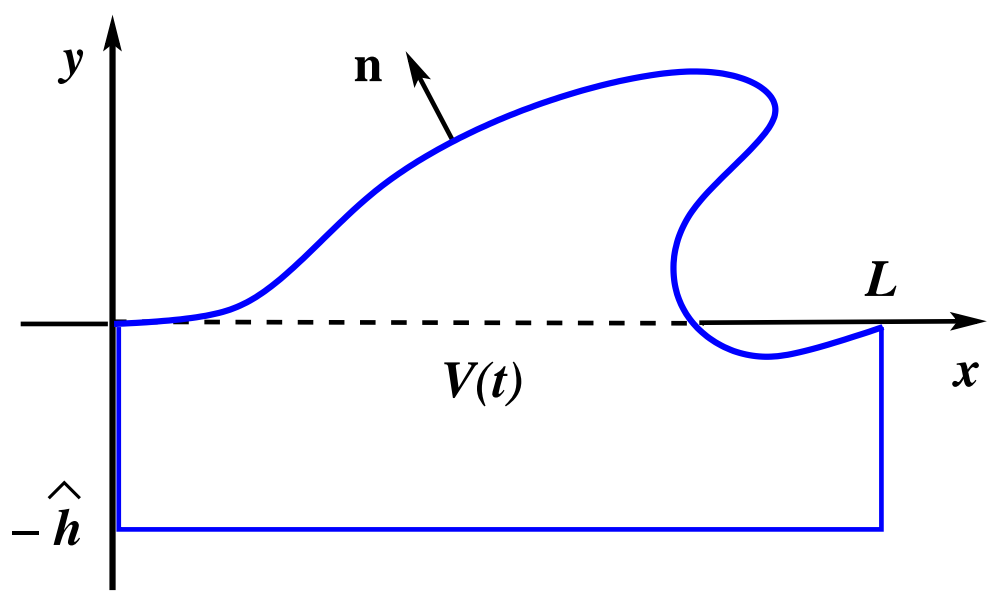

Figure 2: Schematic of the fluid region with free surface.

region occupied by fluid is denoted by $V(t) . \mathbf{n}(\mu, t)$ is the unit normal vector to the free surface, pointing out of the fluid,

$$
\mathbf{n}:=\frac{1}{\ell}\left(\begin{array}{c}
-Y_{\mu} \\
X_{\mu}
\end{array}\right)
$$

with $\ell$ defined in (1.6).

The governing equations for water waves, with the assumption that the flow is irrotational, can be obtained from Luke's variational principle with Lagrangian [25]

$$
\mathscr{L}=\int_{t_{1}}^{t_{2}} \int_{V(t)}\left(\phi_{t}+\frac{1}{2}\left(\phi_{x}^{2}+\phi_{y}^{2}\right)+g y\right) \mathrm{d} x \mathrm{~d} y \mathrm{~d} t,
$$

where $\phi(x, y, t)$ is the velocity potential. Actually Luke's variational principle has been established for the case where the free surface is a graph: $y=\eta(x, t)$. However, Luke's variational principle does carry over to a parametrically defined free surface. Indeed, it is a biproduct of the approach presented here.

Under the transformation (1.1), with $t(\tau)=\tau$, Luke's Lagrangian is transformed to

$$
\mathscr{L}=\int_{\tau_{1}}^{\tau_{2}} \int_{-h}^{0} \int_{0}^{L} \mathcal{L}(\phi, x, y) \mathrm{d} \mu \mathrm{d} \nu \mathrm{d} \tau .
$$


with

$$
\begin{aligned}
\mathcal{L}(\phi, x, y):=J \phi_{\tau}+\left(x_{\nu} y_{\tau}-y_{\nu} x_{\tau}\right) \phi_{\mu}+\left(y_{\mu} x_{\tau}-x_{\mu} y_{\tau}\right) \phi_{\nu} & \\
& +\frac{1}{2} J\left(g^{11} \phi_{\mu}^{2}+2 g^{12} \phi_{\mu} \phi_{\nu}+g^{22} \phi_{\nu}^{2}\right)+g y J .
\end{aligned}
$$

The terms $g^{11}, g^{12}$ and $g^{22}$ are coefficients of the contravariant metric tensor and $J$ is the Jacobian (1.4). An advantage of the Lagrangian (1.9) over the original form (1.8) is that the integration is over a fixed rectangle. Hence the variation of $\mathscr{L}$ just involves the variation of $\mathcal{L}$.

An interesting distinction between Luke's Lagrangian in physical coordinates (1.8) and the transformed version $(1.9)$ is that $x(\mu, \nu, \tau)$ and $y(\mu, \nu, \tau)$ can be interpreted as dependent variables. What is the significance of $\delta \mathcal{L} / \delta x$ and $\delta \mathcal{L} / \delta y$ ? At first one might think that these variations produce equations that give a natural definition of the transformation (1.1). However, this is not the case. These variations actually recover a hidden conservation law of the Laplacian that is not often used.

The following identities can be verifed by direct calculation

$$
\begin{aligned}
\frac{\partial}{\partial x}\left(\frac{1}{2}\left(\phi_{x}^{2}-\phi_{y}^{2}\right)\right)+\frac{\partial}{\partial y}\left(\phi_{x} \phi_{y}\right) & =\phi_{x} \Delta \phi \\
\frac{\partial}{\partial x}\left(\phi_{x} \phi_{y}\right)+\frac{\partial}{\partial y}\left(\frac{1}{2}\left(\phi_{y}^{2}-\phi_{x}^{2}\right)\right) & =\phi_{y} \Delta \phi,
\end{aligned}
$$

where $\Delta \phi$ is the Laplacian in $(x, y)$ coordinates. Hence if $\phi$ is harmonic then the right hand sides vanish giving two conservation laws. It is these two conservation laws - in transformed coordinates - that are generated by $\delta \mathcal{L} / \delta x$ and $\delta \mathcal{L} / \delta y$. The verification of this property is given in $\S 7$ and Appendix B.

There is a close connection with $(x, y)$-variations of the transformed Lagrangian (1.9) and inner variations of the physical Lagrangian (1.8). Hence, a variation can be taken of the original functional (1.8) in the directions of $(x, y)$ while they are still independent variables. These inner variations are discussed in [21] starting on page 149, particularly Lemma 1 on page 149 . A nice feature of the transformed Lagrangian is that inner variations become outer variations.

It is shown in $\S 5$ that the first variation of the Lagrangian (1.9) recovers the governing equations for water waves in curvilinear coordinates. A more surprising result is that it generates a Hamiltonian formulation immediately as well: the Benjamin-Olver Hamiltonian formulation [1] is recovered in transformed coordinates. The symplectic form in the Benjamin-Olver formulation is degenerate, but it applies to waves with arbitrary parameterization of the free surface. On the other hand when the free surface is a graph, $y=\eta(x, t)$, then the symplectic form is canonical, and the Hamiltonian formulation of ZAKHAROV [37] is recovered. ZAKHAROV's Hamiltonian formulation has been extended to curvilinear coordinates by MARCHENKO [26], and our formulation reduces to his when the free surface is represented by a graph.

An outline of the paper is as follows. First the geometric properties of time-dependent transformations of the form (1.1) are outlined. Then the governing equations for water waves in curvilinear coordinates are derived. Then the Lagrangian (1.9) is derived and the first variation taken. In $\S 9$ we briefly discuss particular choices for transformations: explicit transformations based on interpolation, transformations determined by elliptic equations, and the special case of the Lagrangian particle path formulation where the transformation is determined by requiring it to move at the speed of the fluid.

The transformation theory and variational formulations carry over in a straightforward way to three-dimensional waves and some preliminary discussion of this case is in $\S 10$. 


\section{Time-dependent mapping of $V(t)$ to a rectangle}

In this section the geometric properties of the time-dependent transformation (1.1) of $V(t)$ into the fixed rectangle (1.2) are recorded. Taking the differential of (1.1)

$$
\left(\begin{array}{l}
\mathrm{d} x \\
\mathrm{~d} y \\
\mathrm{~d} t
\end{array}\right)=\left[\begin{array}{ccc}
x_{\mu} & x_{\nu} & x_{\tau} \\
y_{\mu} & y_{\nu} & y_{\tau} \\
0 & 0 & t_{\tau}
\end{array}\right]\left(\begin{array}{l}
\mathrm{d} \mu \\
\mathrm{d} \nu \\
\mathrm{d} \tau
\end{array}\right)
$$

The inverse transformation is

$$
\left(\begin{array}{l}
\mathrm{d} \mu \\
\mathrm{d} \nu \\
\mathrm{d} \tau
\end{array}\right)=\frac{1}{J}\left[\begin{array}{ccc}
y_{\nu} & -x_{\nu} & -\tau_{t}\left(y_{\nu} x_{\tau}-x_{\nu} y_{\tau}\right) \\
-y_{\mu} & x_{\mu} & \tau_{t}\left(y_{\mu} x_{\tau}-x_{\mu} y_{\tau}\right) \\
0 & 0 & J_{t}
\end{array}\right]\left(\begin{array}{l}
\mathrm{d} x \\
\mathrm{~d} y \\
\mathrm{~d} t
\end{array}\right):=\left(\begin{array}{ccc}
\mu_{x} & \mu_{y} & \mu_{t} \\
\nu_{x} & \nu_{y} & \nu_{t} \\
\tau_{x} & \tau_{y} & \tau_{t}
\end{array}\right)\left(\begin{array}{l}
\mathrm{d} x \\
\mathrm{~d} y \\
\mathrm{~d} t
\end{array}\right)
$$

The covariant form of the metric associated with this transformation is

$$
\mathrm{d} s^{2}=g_{11} \mathrm{~d} \mu \otimes \mathrm{d} \mu+2 g_{12} \mathrm{~d} \mu \otimes \mathrm{d} \nu+g_{22} \mathrm{~d} \nu \otimes \mathrm{d} \nu,
$$

with the coefficients defined by

$$
\mathbf{G}=\mathbf{M}^{T} \mathbf{M}:=\left(\begin{array}{ll}
g_{11} & g_{12} \\
g_{21} & g_{22}
\end{array}\right), \quad \text { with } \quad \mathbf{M}=\left(\begin{array}{ll}
x_{\mu} & x_{\nu} \\
y_{\mu} & y_{\nu}
\end{array}\right), \quad g_{21}=g_{12}
$$

where

$$
g_{11}=x_{\mu}^{2}+y_{\mu}^{2}, \quad g_{12}=x_{\mu} x_{\nu}+y_{\mu} y_{\nu}, \quad g_{22}=x_{\nu}^{2}+y_{\nu}^{2} .
$$

In the special case of a conformal map, $g_{11}=g_{22}$ and $g_{12}=0$.

The coefficients associated with the contravariant metric are obtained from the inverse

$$
\mathbf{G}^{-1}=\frac{1}{J^{2}}\left[\begin{array}{cc}
g_{22} & -g_{12} \\
-g_{21} & g_{11}
\end{array}\right]:=\left[\begin{array}{ll}
g^{11} & g^{12} \\
g^{21} & g^{22}
\end{array}\right]
$$

using

$$
\operatorname{det}(\mathbf{G})=\operatorname{det}\left(\mathbf{M}^{T} \mathbf{M}\right)=\operatorname{det}(\mathbf{M})^{2}=J^{2} .
$$

Throughout, the time transformation will be limited to $\tau(t)=t$. However, the symbol $\tau$ will be maintained in order to indicate whether the time derivatives are in the physical domain or the transformed domain,

$$
\frac{\partial}{\partial t}:=\left.\frac{\partial}{\partial t}\right|_{(x, y) \text { fixed }} \text { and } \frac{\partial}{\partial \tau}:=\left.\frac{\partial}{\partial t}\right|_{(\mu, \nu) \text { fixed }} .
$$

\subsection{Transformed derivatives and the geometric conservation law}

The transformed spatial derivatives are

$$
\begin{aligned}
& \frac{\partial}{\partial x}=\mu_{x} \frac{\partial}{\partial \mu}+\nu_{x} \frac{\partial}{\partial \nu}=\frac{1}{J} y_{\nu} \frac{\partial}{\partial \mu}-\frac{1}{J} y_{\mu} \frac{\partial}{\partial \nu} \\
& \frac{\partial}{\partial y}=\mu_{y} \frac{\partial}{\partial \mu}+\nu_{y} \frac{\partial}{\partial \nu}=-\frac{1}{J} x_{\nu} \frac{\partial}{\partial \mu}+\frac{1}{J} x_{\mu} \frac{\partial}{\partial \nu},
\end{aligned}
$$

and the transformed time derivative is

$$
\frac{\partial}{\partial t}=\frac{\partial}{\partial \tau}+\mu_{t} \frac{\partial}{\partial \mu}+\nu_{t} \frac{\partial}{\partial \nu}=\frac{\partial}{\partial \tau}+\frac{F_{1}}{J} \frac{\partial}{\partial \mu}+\frac{F_{2}}{J} \frac{\partial}{\partial \nu}
$$


where

$$
F_{1}:=J \mu_{t}=x_{\nu} y_{\tau}-y_{\nu} x_{\tau} \quad \text { and } \quad F_{2}:=J \nu_{t}=y_{\mu} x_{\tau}-x_{\mu} y_{\tau} .
$$

The functions $\left(J, F_{1}, F_{2}\right)$ satisfy the geometric conservation law (GCL)

$$
\frac{\partial J}{\partial \tau}+\frac{\partial F_{1}}{\partial \mu}+\frac{\partial F_{2}}{\partial \nu}=0
$$

This conservation law is trivial in that it is satisfied for any smooth functions of the form $x(\mu, \nu, \tau)$ and $y(\mu, \nu, \tau)$, and it can be verified by direct calculation. The conservation law (2.5) plays a central role in the theory and numerical implementation of moving mesh methods. It was first identified by [31] ((2.5) is a special case of equation (12) in [31]) and is now widely used in moving mesh methods (e.g. [10, 11, 18, 29, 32, 33]).

\section{Governing equations in curvilinear coordinates}

The fluid is assumed to be incompressible (with constant density, normalized to unity), inviscid, and irrotational. For simplicity, surface tension is neglected. The velocity field can be expressed in terms of a potential function $\mathbf{u}:=(u, v)=\nabla \phi$. The governing equations in the physical coordinates $(x, y, t)$ are recorded in Appendix A. In this section a derivation of the governing equations in curvilinear coordinates is presented. For definiteness, periodic boundary conditions are applied at $x=0$ and $x=L$. However, fixed boundaries (such as in the standing-wave or sloshing problem) or other more general boundary domains and conditions can be treated as well.

The potential and velocity field in the transformed coordinates are

$$
\begin{aligned}
& \widehat{\phi}(\mu, \nu, \tau)=\phi(x(\mu, \nu, \tau), y(\mu, \nu, \tau), t(\tau)) \\
& \widehat{u}(\mu, \nu, \tau)=\phi_{x}=\frac{1}{J} y_{\nu} \widehat{\phi}_{\mu}-\frac{1}{J} y_{\mu} \widehat{\phi}_{\nu} \\
& \widehat{v}(\mu, \nu, \tau)=\phi_{y}=-\frac{1}{J} x_{\nu} \widehat{\phi}_{\mu}+\frac{1}{J} x_{\mu} \widehat{\phi}_{\nu}
\end{aligned}
$$

and therefore

$$
\widehat{u}^{2}+\widehat{v}^{2}=\left(g^{11} \widehat{\phi}_{\mu}^{2}+2 g^{12} \widehat{\phi}_{\mu} \widehat{\phi}_{\nu}+g^{22} \widehat{\phi}_{\nu}^{2}\right) .
$$

To avoid excess notation, drop the hats from the transformed variables. It will be clear from the context whether $\phi, u, v$ are relative to the physical coordinates $(x, y, t)$ or the curvilinear coordinates $(\mu, \nu, \tau)$.

The image in $D$ of the time dependent free surface $\Gamma(t)$ is the fixed line $\nu=0$. Take the mapping to limit on the parameterization $\Gamma(t)$ (see $\S 3.2$ for discussion of other parameterizations),

$$
\lim _{\nu \rightarrow 0} x(\mu, \nu, \tau)=X(\mu, \tau) \text { and } \lim _{\nu \rightarrow 0} y(\mu, \nu, \tau)=Y(\mu, \tau) .
$$

Define free surface variables $(\Phi, U, V)$ by

$$
\Phi(\mu, \tau):=\lim _{\nu \rightarrow 0} \phi(\mu, \nu, \tau), \quad U(\mu, \tau):=\lim _{\nu \rightarrow 0} u(\mu, \nu, \tau), \quad V(\mu, \tau):=\lim _{\nu \rightarrow 0} v(\mu, \nu, \tau) .
$$

In the interior of the fluid, Laplace's equation is replaced by the Laplace-Beltrami operator:

$$
\Delta_{g} \phi=0 \text { for }-h<\nu<0 \text { and } 0<\mu<L \text {, }
$$


where

$$
\begin{aligned}
\Delta \phi=u_{x}+v_{y} & =\frac{1}{J}\left(y_{\nu} \frac{\partial u}{\partial \mu}-y_{\mu} \frac{\partial u}{\partial \nu}-x_{\nu} \frac{\partial v}{\partial \mu}+x_{\mu} \frac{\partial v}{\partial \nu}\right) \\
& =\frac{1}{J}\left(\frac{\partial\left(u y_{\nu}\right)}{\partial \mu}-\frac{\partial\left(u y_{\mu}\right)}{\partial \nu}-\frac{\partial\left(v x_{\nu}\right)}{\partial \mu}+\frac{\partial\left(v x_{\mu}\right)}{\partial \nu}\right) \\
& =\frac{1}{J}\left(\frac{\partial\left(u y_{\nu}-v x_{\nu}\right)}{\partial \mu}+\frac{\partial\left(-u y_{\mu}+v x_{\mu}\right)}{\partial \nu}\right) \\
& =\frac{1}{J} \frac{\partial}{\partial \mu}\left(J g^{11} \phi_{\mu}+J g^{12} \phi_{\nu}\right)+\frac{1}{J} \frac{\partial}{\partial \nu}\left(J g^{12} \phi_{\mu}+J g^{22} \phi_{\nu}\right) \\
& :=\Delta_{g} \phi
\end{aligned}
$$

The condition of periodicity (A-3) is replaced by $L$-periodicity in $\mu$. The bottom boundary condition in the transformed coordinates is

$$
v=0 \quad \Rightarrow \quad x_{\mu} \phi_{\nu}-x_{\nu} \phi_{\mu}=0 \quad \text { at } \quad \nu=-h .
$$

In the transformed coordinates the bottom boundary condition is at $\nu=-h$ whereas in the physical coordinates it is at $y=-\widehat{h}$ (cf. equation A-2). In general $h \neq \widehat{h}$. When the transformation is general, $h$ can be chosen to equal to any positive value, but in special cases such as conformal transformations the value of $h$ is constrained by the transformation.

The kinematic free surface boundary condition is the same as (A-7) but with the right-hand side replaced by the normal velocity in transformed coordinates

$$
X_{\mu} Y_{t}-Y_{\mu} X_{t}=-J^{-1} Y_{\mu}\left(y_{\nu} \phi_{\mu}-y_{\mu} \phi_{\nu}\right)+J^{-1} X_{\mu}\left(-x_{\nu} \phi_{\mu}+x_{\mu} \phi_{\nu}\right), \quad \text { at } \nu=0 .
$$

Using boundary velocities (3.2) it is equivalent to

$$
X_{\mu} Y_{t}-Y_{\mu} X_{t}=-U Y_{\mu}+V X_{\mu}, \quad \text { at } \nu=0 .
$$

\subsection{Dynamic free surface boundary condition}

The dynamic free surface boundary condition is a bit more interesting in transformed coordinates and so requires some explanation. Starting with (A-5), and replacing $\phi_{t}$ by the transformed derivative (2.3) and replacing the velocity squared by (3.1), the boundary condition is

$$
\phi_{\tau}+J^{-1} F_{1} \phi_{\mu}+J^{-1} F_{2} \phi_{\nu}+\frac{1}{2}\left(g^{11} \phi_{\mu}^{2}+2 g^{12} \phi_{\mu} \phi_{\nu}+g^{22} \phi_{\nu}^{2}\right)+g Y=0, \quad \text { at } \nu=0 .
$$

The boundary condition (3.7) is expressed relative to an arbitrary transformation of form (1.1). Only special cases have appeared in the literature heretofore. For the case of conformal mapping, where $g_{11}=g_{22}=J$ and $g_{12}=0,(3.7)$ simplifes to

$$
J \phi_{\tau}-\left(Y_{\mu} Y_{\tau}+X_{\mu} X_{\tau}\right) \phi_{\mu}+\left(Y_{\mu} X_{\tau}-X_{\mu} Y_{\tau}\right) \phi_{\nu}+\frac{1}{2}\left(\phi_{\mu}^{2}+\phi_{\nu}^{2}\right)+g Y J=0, \quad \text { at } \nu=0,
$$

and it is this form of the Bernoulli equation (with minor modification) that appears in $[9,13,16$, $22,36]$.

The Bernoulli equation (3.7) arises in the variational formulation in a different form. In the variational formulation it is split into two parts. This splitting is derived from (3.7) as follows.

Define

$$
R:=\phi_{\tau}+\frac{1}{2}\left(g^{11} \phi_{\mu}^{2}+2 g^{12} \phi_{\mu} \phi_{\nu}+g^{22} \phi_{\nu}^{2}\right)+g Y, \quad \text { at } \nu=0 .
$$

Then the dynamic free surface boundary condition (3.7) can be expressed in the form

$$
R+J^{-1} F_{1} \phi_{\mu}+J^{-1} F_{2} \phi_{\nu}=0, \quad \text { at } \nu=0 .
$$


But

$$
\begin{aligned}
J^{-1} F_{1} \phi_{\mu}+J^{-1} F_{2} \phi_{\nu} & =J^{-1}\left(\left(u x_{\mu}+v y_{\mu}\right)\left(x_{\nu} y_{\tau}-y_{\nu} x_{\tau}\right)+\left(u x_{\nu}+v y_{\nu}\right)\left(y_{\mu} x_{\tau}-x_{\mu} y_{\tau}\right)\right) \\
& =J^{-1}\left(-u J x_{\tau}-v J y_{\tau}\right)=-u x_{\tau}-v y_{\tau} .
\end{aligned}
$$

Evaluating at the free surface gives the following form for the boundary condition

$$
R-U X_{\tau}-V Y_{\tau}=0, \quad \text { at } \nu=0 .
$$

Since the surface is non-degenerate, this equation is satisfied if and only if

$$
\mathbf{n}_{j} \cdot\left(R-U X_{\tau}-V Y_{\tau}\right)=0, \quad j=1,2,
$$

where $\mathbf{n}=\left(\mathbf{n}_{1}, \mathbf{n}_{2}\right)$ is the unit normal at the free surface (1.7). Look at the second and third terms in parentheses, and use the kinematic condition and the identity $\Phi_{\mu}=U X_{\mu}+V Y_{\mu}$,

$$
\begin{aligned}
\ell \mathbf{n}_{1} \cdot\left(U X_{\tau}+V Y_{\tau}\right) & =-Y_{\mu}\left(U X_{\tau}+V Y_{\tau}\right) \\
& =-U Y_{\mu} X_{\tau}-Y_{\tau}\left(U X_{\mu}+V Y_{\mu}\right)+U X_{\mu} Y_{\tau} \\
& =-\Phi_{\mu} Y_{\tau}-U^{2} Y_{\mu}+U V X_{\mu} .
\end{aligned}
$$

Similarly

$$
\begin{aligned}
\ell \mathbf{n}_{2} \cdot\left(U X_{\tau}+V Y_{\tau}\right) & =X_{\mu}\left(U X_{\tau}+V Y_{\tau}\right) \\
& =X_{\tau}\left(U X_{\mu}+V Y_{\mu}\right)-V X_{\tau} Y_{\mu}+V X_{\mu} Y_{\tau} \\
& =\Phi_{\mu} X_{\tau}+V^{2} X_{\mu}-U V Y_{\mu} .
\end{aligned}
$$

Substituting these expressions into (3.9) gives

$$
\begin{aligned}
-Y_{\mu} R & =-\Phi_{\mu} Y_{\tau}-U^{2} Y_{\mu}+U V X_{\mu} \\
X_{\mu} R & =\Phi_{\mu} X_{\tau}+V^{2} X_{\mu}-U V Y_{\mu}
\end{aligned}
$$

or

$$
\begin{aligned}
Y_{\mu} \Phi_{\tau}-\Phi_{\mu} Y_{\tau} & =\frac{1}{2}\left(U^{2}-V^{2}\right) Y_{\mu}-U V X_{\mu}-g Y Y_{\mu} \\
-X_{\mu} \Phi_{\tau}+\Phi_{\mu} X_{\tau} & =\frac{1}{2}\left(U^{2}-V^{2}\right) X_{\mu}+U V Y_{\mu}+g Y X_{\mu} .
\end{aligned}
$$

The boundary condition (3.7) is satisfied if and only if both (3.10a) and (3.10b) are satisfied. They are equivalent representations of the dynamic free surface boundary condition.

\subsection{Reparameterization and the free surface representation}

Suppose the free surface is parameterized by a different parameter,

$$
X(\alpha, \tau), \quad Y(\alpha, \tau), \quad 0 \leq \alpha \leq \widehat{L},
$$

for some $\widehat{L}$ which is in general not equal to $L$. In this case

$$
\lim _{\nu \rightarrow 0} x(\mu, \nu, \tau)=X(\alpha, \tau) \text { and } \lim _{\nu \rightarrow 0} y(\mu, \nu, \tau)=Y(\alpha, \tau) .
$$

The curve $(x(\mu, 0, \tau), y(\mu, 0, \tau))$ for each $\tau$ is a reparameterization of the curve (3.11). Therefore there exists a smooth function $\varphi(\alpha, \tau)$ such that $\mu=\varphi(\alpha, \tau)$ with the function $\varphi$ defined by

$$
(x(\varphi(\alpha, \tau), 0, \tau), y(\varphi(\alpha, \tau), 0, \tau))=(X(\alpha, \tau), Y(\alpha, \tau)), \quad \text { for each } \alpha .
$$


Although such a reparameterization looks like an unnecessary complication, there are situations where it is very useful. For example, when the free surface is very steep, a reparameterization is useful in the numerical context to bunch points nearer to the regions of high gradient. In this case the $\alpha$-parameterization would be chosen based on the required mesh for the free surface, and then the reparameterization $\varphi(\alpha, \tau)$ would provide the matching with the interior mapping.

Two examples of a reparameterization of this type in the literature are [34], where a surface reparameterization is introduced in equation (7) on page 2836, and [30], where a surface reparameterization is introduced in equation (6) on page 651. The purpose in both cases was to modify a discretization to bunch grid points near a steep crest. In [20] a time-dependent reparameterization of the surface is implemented using an arclength reparameterization (see $\S 5$ of [20]).

For time-dependent waves the reparameterization would have to be implemented dynamically. Dynamic reparameterization has been used in the moving mesh literature in order to adapt mesh spacing over time (e.g. [10, 11]). For water waves the mesh spacing along the free surface is the most important, and adaptive meshes for one (parameter) space dimension and time are the most effective and well developed. Hence methods such as equidistribution $[10,11,32]$ could be used to determine dynamic mesh spacing at the surface (the $\alpha$-parameterization) and then the interior mapping is just chosen to match smoothly with this parameterization using $\varphi(\alpha, \tau)$. See Chapters 34 and 37 of [33] for extensive discussion of time-dependent adaptive meshing.

\section{Simplifying the density of Luke's Lagrangian}

In this section we study Luke's Lagrangian in transformed coordinates (1.9). The great advantage of the Lagrangian in curvilinear coordinates is that the integration is now over a fixed domain. The density still depends on $\phi, X$ and $Y$, but the new feature is that it explicitly depends on the mapping (1.1). Before considering the first variation of this functional, we show that the time-derivative term and the potential energy term can be simplified dramatically.

\subsection{Simplifying the time derivative term}

In transformed coordinates, $\phi_{t}$ has two representations related by the GCL (2.5)

$$
J \phi_{t}=J \phi_{\tau}+F_{1} \phi_{\mu}+F_{2} \phi_{\nu}=(J \phi)_{\tau}+\left(F_{1} \phi\right)_{\mu}+\left(F_{2} \phi\right)_{\nu}-\phi\left(J_{\tau}+\left(F_{1}\right)_{\mu}+\left(F_{2}\right)_{\nu}\right) .
$$

Hence the integral of the $\phi_{t}$ term in the transformed coordinates can be written as a divergence term in the full 3 -dimensional space-time

$$
\mathscr{T}:=\int_{\tau_{1}}^{\tau_{2}} \int_{-h}^{0} \int_{0}^{L}\left((J \phi)_{\tau}+\left(F_{1} \phi\right)_{\mu}+\left(F_{2} \phi\right)_{\nu}\right) \mathrm{d} \mu \mathrm{d} \nu \mathrm{d} \tau
$$

Hence Green's Theorem can be used to reduce the integral to the 2-dimensional surface of a cuboid of the form

$$
\mathscr{T}=\iint_{S} \phi\left(\begin{array}{c}
F_{1} \\
F_{2} \\
J
\end{array}\right) \cdot \mathcal{N} \mathrm{d} S
$$

where $\mathcal{N}$ is the outward pointing normal on each face of the cuboid.

There are six faces in the cuboid but due to the bottom boundary condition, and the periodicity condition in $\mu$, the integrand is non-zero on only three faces: the faces defined by $t=t_{1}, t=t_{2}$ and $\nu=0$. The terms on the faces $t=t_{1}$ and $t=t_{2}$ are referred to as boundary terms and are

$$
\text { B-terms }:=\left.\int_{-h}^{0} \int_{0}^{L} \Phi J\right|_{t=t_{2}} \mathrm{~d} \mu \mathrm{d} \nu-\left.\int_{-h}^{0} \int_{0}^{L} \Phi J\right|_{t=t_{1}} \mathrm{~d} \mu \mathrm{d} \nu .
$$


The term associated with the face $\nu=0$ is

$$
\left.\int_{\tau_{1}}^{\tau_{2}} \int_{0}^{L}\left(\phi F_{2}\right)\right|_{\nu=0} \mathrm{~d} \nu \mathrm{d} \tau=-\int_{\tau_{1}}^{\tau_{2}} \int_{0}^{L} \Phi\left(Y_{\mu} X_{\tau}-X_{\mu} Y_{\tau}\right) \mathrm{d} \mu \mathrm{d} \tau
$$

since $\mathcal{N}$ on this face is $(0,1,0)$. Hence the integral of $\phi_{t}$ reduces purely to a boundary term

$$
\mathscr{T}=-\int_{\tau_{1}}^{\tau_{2}} \int_{0}^{L} \Phi\left(Y_{\mu} X_{\tau}-X_{\mu} Y_{\tau}\right) \mathrm{d} \mu \mathrm{d} \tau+\text { B-terms } .
$$

\subsection{Potential energy term}

The potential energy term is

$$
\int_{\tau_{1}}^{\tau_{2}} \int_{-h}^{0} \int_{0}^{L} g y\left(x_{\mu} y_{\nu}-x_{\nu} y_{\mu}\right) \mathrm{d} \mu \mathrm{d} \nu \mathrm{d} \tau=\int_{\tau_{1}}^{\tau_{2}} \int_{-h}^{0} \int_{0}^{L}\left[\frac{1}{2} g\left(x_{\mu} y^{2}\right)_{\nu}-\frac{1}{2}\left(x_{\nu} y^{2}\right)_{\mu}\right] \mathrm{d} \mu \mathrm{d} \nu \mathrm{d} \tau .
$$

The integrand is in divergence form in the $(\mu, \nu)$ space only, and hence can be reduced to an integral over the boundary of $D$. With periodic boundary conditions there are only two terms: at $\nu=-h$ and at $\nu=0$

$$
\left.\int_{\tau_{1}}^{\tau_{2}} \int_{0}^{L} \frac{1}{2} g x_{\mu} y^{2}\right|^{\nu=0} \mathrm{~d} \mu \mathrm{d} \tau-\left.\int_{\tau_{1}}^{\tau_{2}} \int_{0}^{L} \frac{1}{2} g x_{\mu} y^{2}\right|_{\nu=-h} \mathrm{~d} \mu \mathrm{d} \tau
$$

However the second term reduces to

$$
\left.\int_{\tau_{1}}^{\tau_{2}} \int_{0}^{L} \frac{1}{2} g x_{\mu} y^{2}\right|_{\nu=-h} \mathrm{~d} \mu \mathrm{d} \tau=\left.\frac{1}{2} g \widehat{h}^{2} \int_{\tau_{1}}^{\tau_{2}} \int_{0}^{L} x_{\mu}\right|_{\nu=-h} \mathrm{~d} \mu \mathrm{d} \tau=\frac{1}{2} g \widehat{h}^{2} L\left(\tau_{2}-\tau_{1}\right),
$$

using the fact that $y=-\widehat{h}$ at $\nu=-h$. This term does not make any contribution to the EulerLagrange equation and can therefore be neglected, reducing the potential energy term to

$$
\int_{\tau_{1}}^{\tau_{2}} \int_{0}^{L} \frac{1}{2} g Y^{2} X_{\mu} \mathrm{d} \mu \mathrm{d} \tau
$$

\subsection{From Luke's Lagrangian to Hamilton's principle}

Substitute the new form of the time derivative term (4.1) and the transformed potential energy (4.2) into Luke's Lagrangian (1.9), neglecting boundary terms,

$$
\mathscr{L}=\int_{\tau_{1}}^{\tau_{2}} \mathcal{L}(\phi, x, y) \mathrm{d} \tau,
$$

with

$$
\mathcal{L}(\phi, x, y)=H(\phi, x, y)-\int_{0}^{L} \Phi\left(X_{\mu} Y_{\tau}-Y_{\mu} X_{\tau}\right) \mathrm{d} \mu
$$

and

$$
H(\phi, x, y)=\int_{-h}^{0} \int_{0}^{L} \frac{1}{2}\left(g^{11} \phi_{\mu}^{2}+2 g^{12} \phi_{\mu} \phi_{\nu}+g^{22} \phi_{\nu}^{2}\right) J \mathrm{~d} \mu \mathrm{d} \nu+\int_{0}^{L} \frac{1}{2} g Y^{2} X_{\mu} \mathrm{d} \mu .
$$

When variations of the Lagrangian with fixed endpoints at $t=t_{1}$ and $t=t_{2}$ are used, the contribution from the B-terms will vanish. Hence for standard variations (as used in this paper) these terms can be neglected. If more general variations are considered, then these terms can be easily restored.

The integrand in (4.4) is in the form of Hamilton's principle (energy minus action). The significance of Hamilton's principle is that the first variation (the Euler-Lagrange equation) of $\mathscr{L}$ will result in a Hamiltonian formulation. 


\section{$5 \quad$ First variation of $\mathscr{L}$}

Take the first variation of $\mathscr{L}$ in $(4.3)$

$$
\delta \mathscr{L}=\int_{\tau_{1}}^{\tau_{2}} \delta \mathcal{L} \mathrm{d} \tau+\{\text { terms at } \mu=0, \mu=L \text { and } \nu=-h\}
$$

with

$$
\delta \mathcal{L}=\int_{-h}^{0} \int_{0}^{L}\left(\frac{\delta \mathcal{L}}{\delta \phi} \delta \phi+\frac{\delta \mathcal{L}}{\delta x} \delta x+\frac{\delta \mathcal{L}}{\delta y} \delta y\right) \mathrm{d} \mu \mathrm{d} \nu+\int_{0}^{L}\left(\frac{\delta \mathcal{L}}{\delta \Phi} \delta \Phi+\frac{\delta \mathcal{L}}{\delta X} \delta X+\frac{\delta \mathcal{L}}{\delta Y} \delta Y\right) \mathrm{d} \mu .
$$

The variations associated with the "terms at $\mu=0, \mu=L$ and $\nu=-h$ " are similiar to the case in physical coordinates and so are only treated briefly (see Appendix $\mathrm{C}$ )

The first variation $\delta \mathcal{L}$ should recover the governing equations for water waves in curvilinear coordinates presented in $\S 3$. However, how the variations produce these equations, as well as additional information, has some interesting features. The main new features are the due to the variations $\delta \mathcal{L} / \delta x$ and $\delta \mathcal{L} / \delta y$. First a summary of the Euler-Lagrange equations is given. The interior derivations depend only on the kinetic energy,

$$
\frac{\delta \mathcal{L}}{\delta \phi}=\frac{\delta \mathcal{K}}{\delta \phi}, \quad \frac{\delta \mathcal{L}}{\delta x}=\frac{\delta \mathcal{K}}{\delta x}, \quad \frac{\delta \mathcal{L}}{\delta y}=\frac{\delta \mathcal{K}}{\delta y} .
$$

where

$$
\mathcal{K}=\frac{1}{2} J\left(g^{11} \phi_{\mu}^{2}+2 g^{12} \phi_{\mu} \phi_{\nu}+g^{22} \phi_{\nu}^{2}\right) .
$$

The variations at the free surface are

$$
\begin{aligned}
\frac{\delta \mathcal{L}}{\delta \Phi} & =\frac{\delta \mathcal{K}}{\delta \Phi}-X_{\mu} Y_{\tau}+X_{\tau} Y_{\mu} \\
\frac{\delta \mathcal{L}}{\delta X} & =\frac{\delta \mathcal{K}}{\delta X}-g Y Y_{\mu}+\Phi_{\mu} Y_{\tau}-\Phi_{\tau} Y_{\mu} \\
\frac{\delta \mathcal{L}}{\delta Y} & =\frac{\delta \mathcal{K}}{\delta Y}+g Y X_{\mu}+\Phi_{\tau} X_{\mu}-\Phi_{\mu} X_{\tau} .
\end{aligned}
$$

Clearly the variation of the kinetic energy is the most important term. The interior variations of $\mathcal{K}$ are

$$
\begin{aligned}
& \frac{\delta \mathcal{K}}{\delta \phi}=-J \Delta_{g} \phi \\
& \frac{\delta \mathcal{K}}{\delta x}=\left(E_{11}\right)_{\mu}+\left(E_{12}\right)_{\nu} \\
& \frac{\delta \mathcal{K}}{\delta y}=\left(E_{21}\right)_{\mu}+\left(E_{22}\right)_{\nu} .
\end{aligned}
$$

The terms $E_{11}, E_{12}, E_{21}$ and $E_{22}$ are the components of the conservation laws (1.11) in the transformed coordinates, and precise expressions are given below in $\S 7$. The variations of the kinetic energy at the surface are

$$
\begin{aligned}
\frac{\delta \mathcal{K}}{\delta \Phi} & =\left.\left(J g^{12} \phi_{\mu}+J g^{22} \phi_{\nu}\right)\right|^{\nu=0} \\
\frac{\delta \mathcal{K}}{\delta X} & =\frac{1}{2}\left(U^{2}-V^{2}\right) Y_{\mu}-U V X_{\mu} \\
\frac{\delta \mathcal{K}}{\delta Y} & =\frac{1}{2}\left(U^{2}-V^{2}\right) X_{\mu}+U V Y_{\mu}
\end{aligned}
$$


These variations (along with the boundary conditions in Appendix C) recover the governing equations for water waves in curvilinear coordinates: $\delta \mathcal{L} / \delta \Phi=0$ recovers the kinematic free surface boundary condition and $\delta \mathcal{L} / \delta X=0$ and $\delta \mathcal{L} / \delta Y=0$ combine to recover the dynamic free surface boundary condition. First, the details of these variations are given, and then the Hamiltonian structure and other properties will be highlighted.

\section{Variation of the kinetic energy with respect to $\phi$}

Define

$$
\mathscr{K}=\int_{\mathscr{V}} \mathcal{K} \mathrm{d} \mu \mathrm{d} \nu
$$

where notation has been simplified by taking

$$
\int_{-h}^{0} \int_{0}^{L} \cdot \mathrm{d} \mu \mathrm{d} \nu:=\int_{\mathscr{V}} \cdot \mathrm{d} \mu \mathrm{d} \nu
$$

The variation of the kinetic energy with respect to $\phi$, with $x$ and $y$ fixed, is

$$
\delta \mathscr{K}=\int_{\mathscr{V}}\left[J g^{11} \phi_{\mu} \delta \phi_{\mu}+J g^{12} \phi_{\nu} \delta \phi_{\mu}+J g^{12} \phi_{\mu} \delta \phi_{\nu}+J g^{22} \phi_{\nu} \delta \phi_{\nu}\right] \mathrm{d} \mu \mathrm{d} \nu,
$$

Integrating by parts reduces the variation to

$$
\delta \mathscr{K}=-\int_{\mathscr{V}} \Delta_{g} \phi \delta \phi J \mathrm{~d} \mu \mathrm{d} \nu+\int_{\mathscr{V}}\left[\frac{\partial P_{1}}{\partial \mu}+\frac{\partial P_{2}}{\partial \nu}\right] \mathrm{d} \mu \mathrm{d} \nu,
$$

where

$$
P_{1}=\left(J g^{11} \phi_{\mu}+J g^{12} \phi_{\nu}\right) \delta \phi \quad \text { and } \quad P_{2}=\left(J g^{12} \phi_{\mu}+J g^{22} \phi_{\nu}\right) \delta \phi .
$$

The first term in (6.1) gives

$$
\frac{\delta \mathcal{K}}{\delta \phi}=-J \Delta_{g} \phi
$$

confirming the first term in (5.4). The second integral in (6.1) can be reduced to a boundary term using Green's theorem. Applying the periodic boundary conditions in the $\mu$-direction, it reduces to an integral over the upper and lower boundaries

$$
\int_{\mathscr{V}}\left[\frac{\partial P_{1}}{\partial \mu}+\frac{\partial P_{2}}{\partial \nu}\right] \mathrm{d} \mu \mathrm{d} \nu=\int_{0}^{L} P_{2}(\mu, 0, \tau) \mathrm{d} \mu-\int_{0}^{L} P_{2}(\mu,-h, \tau) \mathrm{d} \mu .
$$

The term at $\nu=-h$ is considered in Appendix C. The integral at the upper boundary is

$$
\int_{0}^{L} P_{2}(\mu, 0, \tau) \mathrm{d} \mu=\left.\int_{0}^{L}\left(J g^{12} \phi_{\mu}+J g^{22} \phi_{\nu}\right)\right|^{\nu=0} \delta \Phi \mathrm{d} \mu,
$$

hence

$$
\frac{\delta \mathscr{K}}{\delta \Phi}=\left.\left(J g^{12} \phi_{\mu}+J g^{22} \phi_{\nu}\right)\right|^{\nu=0}
$$

verifying the first term in (5.5). By noting that $\ell={\sqrt{g_{11}}}^{\nu=0}$ and modifying the inner product, the integral can be written

$$
\int_{0}^{L} P_{2}(\mu, 0, \tau) \mathrm{d} \mu=\left.\int_{0}^{L} \frac{1}{\sqrt{g_{11}}}\left(J g^{12} \phi_{\mu}+J g^{22} \phi_{\nu}\right)\right|^{\nu=0} \delta \Phi \ell \mathrm{d} \mu
$$

giving

$$
\frac{\delta \mathscr{K}}{\delta \Phi}=\left.\frac{1}{\sqrt{g_{11}}}\left(J g^{12} \phi_{\mu}+J g^{22} \phi_{\nu}\right)\right|^{\nu=0}=\left.\mathbf{u} \cdot \mathbf{n}\right|^{\nu=0},
$$

recovering the familiar result that the variation of Luke's Lagrangian in the direction of $\Phi$ gives the normal velocity at the surface. 


\section{$7 \quad$ Variation of the kinetic energy with respect to $x$ and $y$}

The variation of the Lagrangian with respect to $x$ and $y$ is a new feature of the transformed

Lagrangian. In this section the details of the variation of $\mathscr{K}$ with respect to $x$ and $y$ with $\phi$ fixed are presented. Start with

$$
\begin{aligned}
\delta \mathscr{K}= & \int_{\mathscr{V}}\left[-\frac{1}{2} q^{2}\left[x_{\mu} \delta y_{\nu}+\delta x_{\mu} y_{\nu}-\delta x_{\nu} y_{\mu}-x_{\nu} \delta y_{\mu}\right]\right. \\
& +\frac{1}{J} \phi_{\mu}^{2}\left[x_{\nu} \delta x_{\nu}+y_{\nu} \delta y_{\nu}\right]+\frac{1}{J} \phi_{\nu}^{2}\left[x_{\mu} \delta x_{\mu}+y_{\mu} \delta y_{\mu}\right] \\
& \left.-\frac{1}{J} \phi_{\mu} \phi_{\nu}\left[x_{\mu} \delta x_{\nu}+x_{\nu} \delta x_{\mu}+y_{\mu} \delta y_{\nu}+y_{\nu} \delta y_{\mu}\right]\right] \mathrm{d} \mu \mathrm{d} \nu
\end{aligned}
$$

After some algebra, this variation can be reduced to

$$
\delta \mathscr{K}=\int_{\mathscr{V}}\left[E_{1} \delta x+E_{2} \delta y+\frac{\partial e_{1}}{\partial \mu}+\frac{\partial e_{2}}{\partial \nu}\right] \mathrm{d} \mu \mathrm{d} \nu,
$$

where

$$
\begin{aligned}
& e_{1}=-\frac{1}{2} q^{2} y_{\nu} \delta x+\frac{1}{2} q^{2} x_{\nu} \delta y+\phi_{\nu}\left[\frac{x_{\mu}}{J} \phi_{\nu}-\frac{x_{\nu}}{J} \phi_{\mu}\right] \delta x+\phi_{\nu}\left[\frac{y_{\mu}}{J} \phi_{\nu}-\frac{y_{\nu}}{J} \phi_{\mu}\right] \delta y \\
& e_{2}=-\frac{1}{2} q^{2} x_{\mu} \delta y+\frac{1}{2} q^{2} y_{\mu} \delta x+\phi_{\mu}\left[\frac{x_{\nu}}{J} \phi_{\mu}-\frac{x_{\mu}}{J} \phi_{\nu}\right] \delta x+\phi_{\mu}\left[\frac{y_{\nu}}{J} \phi_{\mu}-\frac{y_{\mu}}{J} \phi_{\nu}\right] \delta y
\end{aligned}
$$

and

$$
\begin{aligned}
& E_{1}=\left(E_{11}\right)_{\mu}+\left(E_{12}\right)_{\nu} \\
& E_{2}=\left(E_{21}\right)_{\mu}+\left(E_{22}\right)_{\nu},
\end{aligned}
$$

with

$$
\begin{aligned}
& E_{11}=\frac{1}{2} q^{2} y_{\nu}-\phi_{\nu}\left[\frac{x_{\mu}}{J} \phi_{\nu}-\frac{x_{\nu}}{J} \phi_{\mu}\right], \quad E_{12}=-\frac{1}{2} q^{2} y_{\mu}-\phi_{\mu}\left[\frac{x_{\nu}}{J} \phi_{\mu}-\frac{x_{\mu}}{J} \phi_{\nu}\right] \\
& E_{21}=-\frac{1}{2} q^{2} x_{\nu}-\phi_{\nu}\left[\frac{y_{\mu}}{J} \phi_{\nu}-\frac{y_{\nu}}{J} \phi_{\mu}\right], \quad E_{22}=\frac{1}{2} q^{2} x_{\mu}-\phi_{\mu}\left[\frac{y_{\nu}}{J} \phi_{\mu}-\frac{y_{\mu}}{J} \phi_{\nu}\right] .
\end{aligned}
$$

It is immediate that

$$
\frac{\delta \mathcal{K}}{\delta x}=E_{1}=\left(E_{11}\right)_{\mu}+\left(E_{12}\right)_{\nu}, \quad \frac{\delta \mathcal{K}}{\delta y}=E_{2}=\left(E_{21}\right)_{\mu}+\left(E_{22}\right)_{\nu} .
$$

To determine the significance of these equations first rewrite the components in a form that highlights the velocity

$$
\begin{aligned}
E_{11} & =\frac{1}{2} q^{2} y_{\nu}-\phi_{\nu}\left[\frac{x_{\mu}}{J} \phi_{\nu}-\frac{x_{\nu}}{J} \phi_{\mu}\right] \\
& =\frac{1}{2}\left(u^{2}+v^{2}\right) y_{\nu}-v \phi_{\nu} \\
& =\frac{1}{2}\left(u^{2}-v^{2}\right) y_{\nu}+v^{2} y_{\nu}-v\left(u x_{\nu}+v y_{\nu}\right) \\
& =\frac{1}{2}\left(u^{2}-v^{2}\right) y_{\nu}-u v x_{\nu} .
\end{aligned}
$$

Similarly for the other three terms

$$
\begin{aligned}
& E_{12}=u v x_{\mu}-\frac{1}{2}\left(u^{2}-v^{2}\right) y_{\mu} \\
& E_{21}=\frac{1}{2}\left(u^{2}-v^{2}\right) x_{\nu}+u v y_{\nu} \\
& E_{22}=-\frac{1}{2}\left(u^{2}-v^{2}\right) x_{\mu}-u v y_{\mu} .
\end{aligned}
$$

In Appendix B the conservation laws (1.11) are transformed to a conservation law in $(\mu, \nu)$ - coordinates. Comparison of the conservation laws in Appendix B with the above expressions confirms that setting the variation of $\mathscr{L}$ with respect to $x$ and $y$ to zero recovers precisely the conservation laws (1.11). 


\subsection{Variation with respect to $X$ and $Y$ at the surface}

The divergence term $\left(e_{1}\right)_{\mu}+\left(e_{2}\right)_{\nu}$ in the variation (7.1) can be reduced to an integral over the boundary of $D$ using Green's theorem. The terms at $\mu=0$ and $\mu=L$ vanish due to periodicity, leaving

$$
\int_{\mathscr{V}}\left[\frac{\partial e_{1}}{\partial \mu}+\frac{\partial e_{2}}{\partial \nu}\right] \mathrm{d} \mu \mathrm{d} \nu=\int_{0}^{L} e_{2}(\mu, 0, \tau) \mathrm{d} \mu-\int_{0}^{L} e_{2}(\mu,-h, \tau) \mathrm{d} \mu .
$$

But $\left.e_{2}\right|_{\nu=-h}=0$ since $v=0$ and $y(\mu, 0, \tau)$ is constant, leaving

$$
\int_{\mathscr{V}}\left[\frac{\partial e_{1}}{\partial \mu}+\frac{\partial e_{2}}{\partial \nu}\right] \mathrm{d} \mu \mathrm{d} \nu=\int_{0}^{L} e_{2}(\mu, 0, \tau) \mathrm{d} \mu .
$$

At $\nu=0, x=X, y=Y$ and $\phi=\Phi$ and so

$$
\left.e_{2}\right|^{\nu=0}=-\frac{1}{2} q^{2} X_{\mu} \delta Y+\frac{1}{2} q^{2} Y_{\mu} \delta X+\Phi_{\mu}\left[\frac{x_{\nu}}{J} \phi_{\mu}-\frac{x_{\mu}}{J} \phi_{\nu}\right] \delta X+\Phi_{\mu}\left[\frac{y_{\nu}}{J} \phi_{\mu}-\frac{y_{\mu}}{J} \phi_{\nu}\right] \delta Y .
$$

Expressed in terms of the surface velocities this is

$$
\left.e_{2}\right|^{\nu=0}=\left[\frac{1}{2}\left(U^{2}-V^{2}\right) Y_{\mu}-U V X_{\mu}\right] \delta X+\left[\frac{1}{2}\left(U^{2}-V^{2}\right) X_{\mu}+U V Y_{\mu}\right] \delta Y,
$$

confirming that

$$
\frac{\delta \mathcal{K}}{\delta X}=\frac{1}{2}\left(U^{2}-V^{2}\right) Y_{\mu}-U V X_{\mu} \quad \text { and } \quad \frac{\delta \mathcal{K}}{\delta Y}=\frac{1}{2}\left(U^{2}-V^{2}\right) X_{\mu}+U V Y_{\mu}
$$

\section{Hamiltonian formulation in curvilinear coordinates}

The three equations at the free surface generated by the first variation of (1.9), given in (5.3), can be written in the form

$$
\left[\begin{array}{ccc}
0 & -\Phi_{\mu} & Y_{\mu} \\
\Phi_{\mu} & 0 & -X_{\mu} \\
-Y_{\mu} & X_{\mu} & 0
\end{array}\right]\left(\begin{array}{l}
X \\
Y \\
\Phi
\end{array}\right)_{\tau}=\left(\begin{array}{c}
\delta H / \delta X \\
\delta H / \delta Y \\
\delta H / \delta \Phi
\end{array}\right)
$$

The left-hand side looks very similar to the BenJamin-Olver [1] formulation (see also $\S 4.5$ of [2] and $\S 2.5$ of [15]). The difference is that $\Phi$ here is evaluated in the transformed coordinates on the line $\nu=0$ whereas in [1] $\Phi$ is evaluated at free surface in the physical domain. In the formulation

[1], the right-hand side of (8.1) is quite complicated. Here the expressions in curvilinear coordinates simplify to

$$
\begin{aligned}
& \frac{\delta H}{\delta X}=\frac{1}{2}\left(U^{2}-V^{2}\right) Y_{\mu}-U V X_{\mu}-g Y Y_{\mu} \\
& \frac{\delta H}{\delta Y}=\frac{1}{2}\left(U^{2}-V^{2}\right) X_{\mu}+U V Y_{\mu}+g Y X_{\mu} \\
& \frac{\delta H}{\delta \Phi}=-Y_{\mu} U+X_{\mu} V .
\end{aligned}
$$

The equation (8.1) can be written in another illuminating form,

$$
\mathbf{Z}_{\mu} \times \mathbf{Z}_{\tau}=\nabla H(\mathbf{Z})
$$

where $\mathrm{x}$ is the standard cross product and $\mathbf{Z}=(X, Y, \Phi)$. The symplectic operator associated with the left-hand side of (8.1) is

$$
\Omega=\int_{0}^{L} \Phi\left(X_{\mu} \mathrm{d} Y-\Phi Y_{\mu} \mathrm{d} X\right) \mathrm{d} \mu .
$$


A proof that this differential form is closed and hence generates a symplectic structure follows the same lines as the proof in $\S 4.4$ of [2]. However, the symplectic structure is degenerate. The kernel of the matrix operator on the left-hand side of (8.1) is spanned by $\mathbf{Z}_{\mu}$. This degeneracy is due to the reparameterization symmetry. This degeneracy may be useful in numerics, when introducing a dynamic reparameterization of the surface as in $\S 3.2$.

\subsection{Zakharov's Hamiltonian formulation in curvilinear coordinates}

Replacing the parametric formulation of the free surface by a graph representation, $y=\eta(x, t)$, the free surface in curvilinear coordinates has the constraint

$$
y(\mu, 0, \tau)=\eta(x(\mu, 0, \tau), \tau) .
$$

This representation is cumbersome as the graph function $\eta$ needs to be carried along in the formulation, whereas the parameterically defined free surface (1.5) matches the transformation (1.1) neatly. Nevertheless one can still construct a Hamiltonian formulation. MARCHENKo [26] extends Zakharov's Hamiltonian formulation to the case of curvilinear coordinates for this case. The Hamiltonian formulation in [26] is recovered by replacing the parametrically-defined free surface by the graph constraint (8.3) and revising the density for Hamilton's principle (4.4). Taking the first variation then recovers equation (2.3) in [26].

\section{Particular classes of transformation}

There are a number of different ways that the mapping (1.1) can be generated. The simplest method is to specify the transformation explicitly. A general method for this approach is to use transfinite interpolation, where the boundary is mapped exactly and then the interior points of the mapping are determined by interpolation. A more powerful method is to generate the mapping as the solution of an elliptic PDE. A special case of this approach is conformal mapping. Another approach is to have the mapping move precisely at the speed of the fluid:

$$
x_{\tau}=u \quad \text { and } \quad y_{\tau}=v .
$$

This case recovers the Lagrangian particle path (LPP) formulation of fluid dynamics. In the context of this paper it recovers the LPP formulation with the additional assumption of irrotationality.

\subsection{Transfinite interpolation}

Transfinite interpolation (TFI) is the simplest way to map a quadrilateral in the $(x, y)$ plane into a rectangle in the $(\mu, \nu)$ plane. The methodology is described in $\S 4.3$ of [18].

Let $\mathbf{r}(\mu, \nu)$ be the mapping taking the unit square in the $(\mu, \nu)$ plane into the quadrilateral in the $(x, y)$ plane as shown schematically in Figure 3. It is constructed as follows. Parameterize the four segments of the quadrilateral in the $(x, y)$ plane

$$
\begin{array}{cll}
A B: & \mathbf{r}_{l}(\nu)=\mathbf{r}(0, \nu) & 0 \leq \nu \leq 1 \\
B C: & \mathbf{r}_{t}(\mu)=\mathbf{r}(\mu, 1) & 0 \leq \mu \leq 1 \\
D C: & \mathbf{r}_{r}(\nu)=\mathbf{r}(1, \nu) & 0 \leq \nu \leq 1 \\
A D: & \mathbf{r}_{b}(\mu)=\mathbf{r}(\mu, 0) & 0 \leq \mu \leq 1 .
\end{array}
$$

The parameterized curves $r_{l}(\nu), r_{t}(\mu), r_{r}(\nu), r_{b}(\nu)$ are assumed to be given. The above specification assures that the boundary of the quadrilateral is mapped to the edges of the square in the 


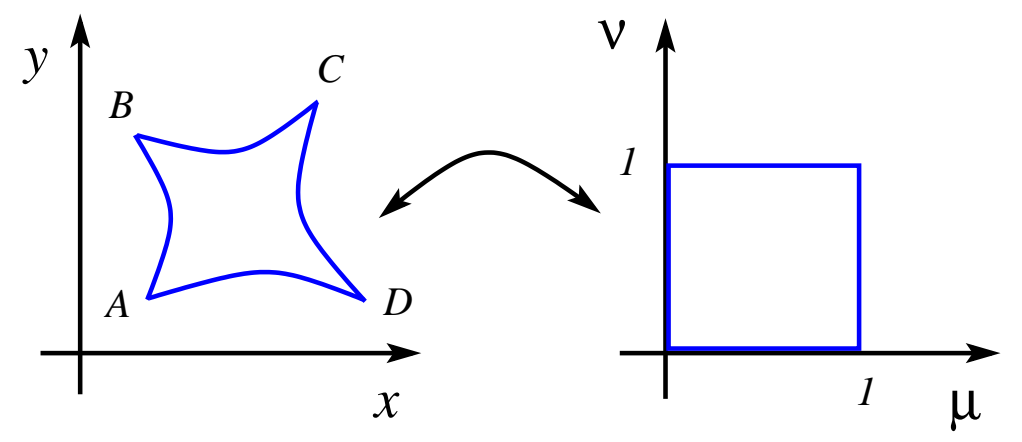

Figure 3: Schematic of transfinite interpolation.

$(\mu, \nu)$ plane. TFI is a method for interpolating between the four boundaries. Let

$$
\begin{aligned}
& \mathbf{R}_{\mu}(\mu, \nu)=(1-\mu) \mathbf{r}(0, \nu)+\mu \mathbf{r}(1, \nu) \\
& \mathbf{R}_{\nu}(\mu, \nu)=(1-\nu) \mathbf{r}(\mu, 0)+\nu \mathbf{r}(\mu, 1) .
\end{aligned}
$$

The formula for transfinite interpolation is then

$$
\mathbf{r}(\mu, \nu)=\mathbf{R}_{\mu}(\mu, \nu)+\mathbf{R}_{\nu}(\mu, \nu)-\mathbf{R}_{\mu}(\mu, \nu) \circ \mathbf{R}_{\nu}(\mu, \nu),
$$

where

$$
\mathbf{R}_{\mu}(\mu, \nu) \circ \mathbf{R}_{\nu}(\mu, \nu)=(1-\mu)(1-\nu) \mathbf{r}(0,0)+(1-\mu) \nu \mathbf{r}(0,1)+\mu(1-\nu) \mathbf{r}(1,0)+\mu \nu \mathbf{r}(1,1) .
$$

Apply to the case of water waves to map $V(t)$ into $D$. In this case

$$
\begin{aligned}
\mathbf{r}_{l}(\nu, \tau) & =(0, \nu) \quad-h \leq \nu \leq 0 \\
\mathbf{r}_{t}(\mu, \tau) & =(X(\mu, \tau), Y(\mu, \tau)) \quad 0 \leq \mu \leq L \\
\mathbf{r}_{r}(\nu, \tau) & =(L, \nu) \quad-h \leq \nu \leq 0 \\
\mathbf{r}_{b}(\mu, \tau) & =(\mu,-h) \quad 0 \leq \mu \leq L
\end{aligned}
$$

Substituting into the formula (9.1) gives

$$
\begin{aligned}
& x(\mu, \nu, \tau)=\mu+\frac{(\nu+h)}{h}(X(\mu, \tau)-\mu) \\
& y(\mu, \nu, \tau)=\frac{(\nu+h)}{h} Y(\mu, \tau) .
\end{aligned}
$$

There is however a restriction on the mapping due to the requirement that $J \neq 0$, where

$$
J=-\frac{\nu}{h^{2}} Y+\frac{(\nu+h)}{h^{2}}\left(Y X_{\mu}-X Y_{\mu}\right)+\frac{\mu \nu}{h^{2}} Y_{\mu}
$$

\subsection{Elliptic PDE grid generation}

One of the most widely used methods for constructing the mapping is to determine $x(\mu, \nu, \tau)$ and $y(\mu, \nu, \tau)$ as solutions of an elliptic PDE. The PDEs are first defined in the $(x, y)$ plane

$$
\Delta \mu=f_{1}(\mu, \nu) \text { and } \quad \Delta \nu=f_{2}(\mu, \nu)
$$


where $f_{1}(\mu, \nu)$ and $f_{2}(\mu, \nu)$ are specified control functions and $\Delta$ is the Laplacian in the $(x, y)$ plane. The control functions are used to control the grid spacing, allowing for improved resolution in regions of high gradient.

These equations are inverted to give nonlinear elliptic PDEs in the $(\mu, \nu)$ plane

$$
\begin{aligned}
g^{11} x_{\mu \mu}+2 g^{12} x_{\mu \nu}+g^{22} x_{\nu \nu}+f_{1} x_{\mu}+f_{2} x_{\nu} & =0 \\
g^{11} y_{\mu \mu}+2 g^{12} y_{\mu \nu}+g^{22} y_{\nu \nu}+f_{1} y_{\mu}+f_{2} y_{\nu} & =0 .
\end{aligned}
$$

A derivation is given in Chapter 5 of [18]. In the special case that the mapping is conformal, $f_{1}=f_{2}=0 \quad g_{12}=0$ and $g_{11}=g_{22}$, and these equations just reduce to Laplace's equation. The solution of these PDEs then provides the mapping for each $\tau$.

This method can be adapted to be time dependent. A number of approaches have been suggested. See Chapter 7 of [18], and [33, 32, 31, 29, 10, 11].

\subsection{Time-dependent conformal mapping}

In time-dependent conformal mapping, the mapping is required to satisfy the Cauchy-Riemann equations

$$
x_{\mu}=y_{\nu} \quad \text { and } \quad x_{\nu}=-y_{\mu} .
$$

This approach to mapping is the most widely used in the literature and details can be found in the references (e.g. $[9,13,16,17,22,23,28,35,36])$. Here we will just highlight one of the difficulties with conformal mapping: the conformal modulus. According to the Riemann mapping theorem one can specify at most 3 points on the boundary of the simply connected region. If four points are fixed then the aspect ratio of the rectangle in the $(\mu, \nu)$ plane can not be chosen independently. A schematic of such a mapping is shown in Figure 4. Some elementary examples of the conformal

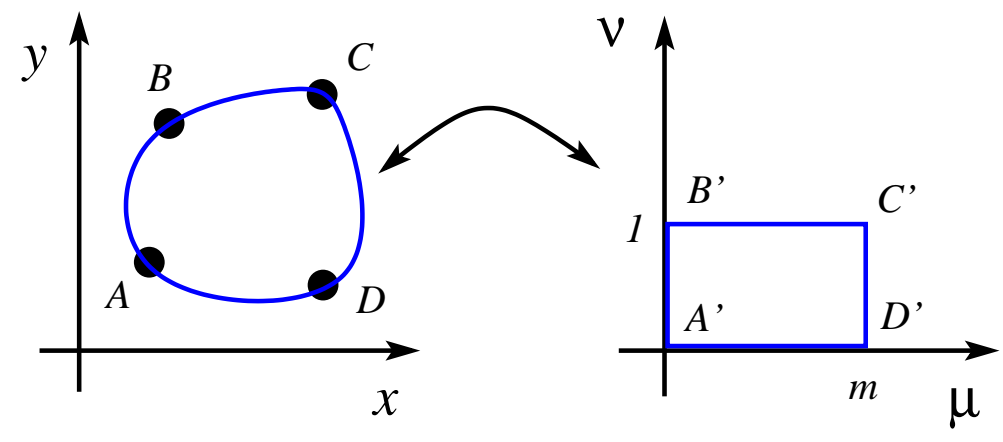

Figure 4: Illustration of the conformal modulus problem.

modulus for various shapes can be found in the papers of Challis \& Burley [12] and SEIDL \& KLOSE [27]. In the time-dependent case, the conformal modulus will depend on time! In general a time-dependent conformal map can not be used to map to a fixed rectangle.

The conformal modulus problem only arises in finite depth. In the case of infinite depth the conformal modulus problem disappears. In the case of infinite depth with periodic boundary conditions, time-dependent conformal mapping is very efficient since the Hilbert transform can be used to determine conjugate harmonic functions, and there is no conformal modulus problem. See [28] for a review of this case and additional references. However, there is still the grid spacing issue which is a problem for very steep waves.

The mapping can be combined with the variational approach by adding the defining conditions into Luke's variational principle as constraints. For example if the mapping is required to be 
conformal, then the Cauchy-Riemann equations (9.2) would be added as constraints using Lagrange multipliers. The Lagrangian functional (4.4) is modified to

$$
\mathscr{L}=\int_{t_{1}}^{t_{2}}\left[H-\int_{0}^{L} \Phi\left(X_{\mu} Y_{t}-Y_{\mu} X_{t}\right) \mathrm{d} \mu-\int_{-h}^{0} \int_{0}^{L}\left(\lambda_{1}\left(x_{\mu}-y_{\nu}\right)-\lambda_{2}\left(x_{\nu}+y_{\mu}\right)\right) \mathrm{d} \mu \mathrm{d} \nu\right] \mathrm{d} t,
$$

with Lagrange multipliers $\lambda_{1}$ and $\lambda_{2}$.

\subsection{Lagrangian particle path formulation}

In the Lagrangian particle path formulation the mapping is determined by requiring the grid to move at the fluid velocity

$$
x_{\tau}=\frac{\left(y_{\nu} \phi_{\mu}-y_{\mu} \phi_{\nu}\right)}{J} \text { and } y_{\tau}=\frac{\left(-x_{\nu} \phi_{\mu}+x_{\mu} \phi_{\nu}\right)}{J} .
$$

It follows from the geometric conservation law (2.5) that $J$ is independent of time. The kinematic condition becomes superfluous, and the dynamic condition reduces to

$$
\phi_{\tau}-\frac{1}{2}\left(x_{\tau}^{2}+y_{\tau}^{2}\right)+g Y=0, \quad \text { at } \quad \nu=0 .
$$

Note the change of sign of the velocity squared term. $\phi$ is determined by solving the Laplace Beltrami operator in the interior, and applying the bottom boundary condition.

With $x_{\tau}$ replaced by $\phi_{x}$ and $y_{\tau}$ replaced by $\phi_{y}$, the equation (9.4) was first derived by Longuet-Higgins \& Cokelet [24] (see equation (2.9) in [24]). However, solving LPP in the irrotational formulation does not appear to be any simpler than solving the LPP formulation with vorticity, since the complete Euler equations in the LPP formulation have a very nice vartiational formulation [8].

\section{Concluding remarks}

In this paper the variational principles for water waves, in the irrotational flow approximation, have been studied under arbitrary smooth transformation to a fixed domain. The motivation for this study is fundamental. A wide range of special transformations have been used in the literature, and it is firstly of interest to study general transformations and itemize those features that are common to all transformations, and secondly to study their impact on variational formulations.

Although the approach in this paper was inspired by the theory of moving grids in computational fluid dynamics, it does not necessarily lead to faster numerical schemes. The choice of numerical method in a given problem will depend on the nature of the questions to be addressed. For example, numerical conformal mapping is still a very powerful approach, in spite of its shortcomings. For periodic boundary conditions, and a free surface without severe distortion, the conformal map with the Hilbert transformation is probably fastest, but the issue with the time-dependent conformal modulus must be addressed in the finite depth case. But for distorted free surfaces, other methods, such as direct use of Fourier series without conformal mapping are more robust (e.g. [20]). The most powerful approach to grid generation is to use grids produced as solutions of PDEs. However, in two space dimensions it is very difficult to argue in favour of this approach for computation as it involves solving additional PDEs. Hence, in two dimensions, use of elliptic PDEs for grid generation is very appealing but would be computationally expensive. Although with parallel computing they may be feasible.

The issue with mappings changes dramatically in the case of three-dimensional waves. The main problem being that conformal mappings are no longer of any use. Hence the use of explicit 
mappings or grid generation using elliptic PDEs are more important. Grid generation using elliptic PDEs could be competitive if parallelization of the 3 PDEs for the mesh is effective.

On the other hand, the basic theory for the interaction between mappings and variational principles extends in a natural way to three-dimensional waves. The $3 \mathrm{D}$ analogue of (1.1) is

$$
(\mu, \nu, \zeta, \tau) \mapsto\left\{\begin{array}{l}
x(\mu, \nu, \zeta, \tau) \\
y(\mu, \nu, \zeta, \tau) \\
z(\mu, \nu, \zeta, \tau) \\
t(\tau)
\end{array} .\right.
$$

The free surface can be parametrically defined by $(X(\mu, \nu, \tau), Y(\mu, \nu, \tau), Z(\mu, \nu, \tau))$ and the transformation can be defined to match in the limit $\zeta \rightarrow 0$,

$$
\left(\begin{array}{l}
X(\mu, \nu, \tau) \\
Y(\mu, \nu, \tau) \\
Z(\mu, \nu, \tau)
\end{array}\right)=\lim _{\zeta \rightarrow 0}\left(\begin{array}{l}
x(\mu, \nu, \zeta, \tau) \\
y(\mu, \nu, \zeta, \tau) \\
z(\mu, \nu, \zeta, \tau)
\end{array}\right)
$$

Luke's Lagrangian has the same form as (1.8) extended to 3D, with $V(t)$ the analogous 3D fluid volume. The only difficult term in the extension to $3 \mathrm{D}$ is the time derivative. Preliminary results show that it is of the form

$$
-\int_{t_{1}}^{t_{2}} \int_{V(t)} \phi_{t} \mathrm{~d} x \mathrm{~d} y \mathrm{~d} z \mathrm{~d} t=\int_{\tau_{1}}^{\tau_{2}} \int_{S} \Phi(\mu, \nu, \tau) \operatorname{det}\left[\begin{array}{ccc}
X_{t} & X_{\mu} & X_{\nu} \\
Y_{t} & Y_{\mu} & Y_{\nu} \\
Z_{t} & Z_{\mu} & Z_{\nu}
\end{array}\right] \mathrm{d} \mu \mathrm{d} \nu \mathrm{d} \tau+\text { B-terms. }
$$

The full details of the $3 \mathrm{D}$ case will be considered elsewhere (e.g. [6]).

One variational approach that we have not considered is the multi-symplectic formulation $[3,4$, 7]. The water wave problem was shown to be multi-symplectic in [3], and in principle the analogue in curvilinear coordinates can be obtained by simply pullback of the defining symplectic forms. This strategy is straightforward if the transformation is specified. However, if the transformation is to be solved for (say as the solution of an elliptic equation), then these equations need to be added to the multi-symplectic structure and so one needs to re-derive the multi-symplectic structure accounting for the variations of the mapping.

\section{Appendix -}

\section{A Governing equations}

In physical coordinates, the principal dependent variables are $\phi(x, y, t)$, the velocity potential, and $(X(\mu, t), Y(\mu, t))$ the parametric representation of the free surface. In this appendix the governing equations for an incompressible, inviscid and irrotational fluid are recorded. The velocity potential $\phi$ satisfys Laplace's equation in the interior

$$
\Delta \phi:=\phi_{x x}+\phi_{y y}=0, \quad \text { for all } \quad(x, y) \in V(t) .
$$

The boundary condition for $\phi$ at the bottom is

$$
\phi_{y}=0 \quad \text { at } \quad y=-\widehat{h} .
$$


Periodic boundary conditions are imposed at $x=0$ and $x=L$

$$
\nabla \phi(x+L, y, t)=\nabla \phi(x, y, t), \quad(x, y) \in V(t) .
$$

The period in $\mu$ space is also taken to be $L$,

$$
X(\mu+L, t)=L+X(\mu, t) \text { and } Y(\mu+L, t)=Y(\mu, t), \quad \text { for all } \quad \mu .
$$

The dynamic free surface boundary condition requires the pressure to be continuous across the surface, which, using the Bernoulli equation, can be expressed in the form

$$
\phi_{t}+\frac{1}{2}\left(\phi_{x}^{2}+\phi_{y}^{2}\right)+g y=0 \quad \text { for } \quad(x, y) \in \Gamma(t),
$$

where the Bernoulli function is set to zero and atmospheric pressure is normalized to be zero.

The kinematic free surface boundary condition requires the normal velocity of the surface to be equal to the normal velocity of the fluid at the surface,

$$
\mathbf{n} \cdot \mathbf{X}_{t}=\mathbf{n} \cdot \nabla \phi, \quad \text { for } \quad(x, y) \in \Gamma(t),
$$

where $\mathbf{X}(\mu, t)=(X(\mu, t), Y(\mu, t))$ and $\mathbf{n}(\mu, t)$ is the unit normal vector to the free surface (1.7). Written out, the kinematic free surface boundary condition is

$$
X_{\mu} Y_{t}-Y_{\mu} X_{t}=-Y_{\mu} \phi_{x}+X_{\mu} \phi_{y}, \quad \text { for } \quad(x, y) \in \Gamma(t) .
$$

In the special case of a single-valued free surface that can be expressed as a graph,

$$
x(\mu, \nu, t):=\mu \quad \text { and } \quad Y(\mu, t):=\eta(x, t),
$$

the kinematic condition reduces to the familiar form

$$
\eta_{t}+\phi_{x} \eta_{x}=\phi_{y}, \quad \text { at } \quad y=\eta(x, t), \quad x \in \mathbb{R} .
$$

\section{B Hidden conservation laws associated with Laplace's equation}

In this appendix the conservation laws (1.11) are transformed to conservation laws in the $(\mu, \nu)-$ coordinates. Let $A=\frac{1}{2}\left(\phi_{x}^{2}-\phi_{y}^{2}\right)$ and $B=\phi_{x} \phi_{y}$. Then the first conservation law in (1.11) is $A_{x}+B_{y}=0$. The simplest way to transform conservation laws to new coordinates is to use differential forms [5]. Let

$$
\Omega_{1}=A \mathrm{~d} y-B \mathrm{~d} x,
$$

then

$$
\mathrm{d} \Omega_{1}=\left(A_{x}+B_{y}\right) \mathrm{d} x \wedge \mathrm{d} y,
$$

and so $A_{x}+B_{y}=0$ is equivalent to $\mathrm{d} \Omega_{1}=0$.

Pullback the $(x, y)$-coordinates to the $(\mu, \nu)$-coordinates,

$$
\Omega_{1}=A\left(y_{\nu} \mathrm{d} \nu+y_{\mu} \mathrm{d} \mu\right)-B\left(x_{\nu} \mathrm{d} \nu+x_{\mu} \mathrm{d} \mu\right)=\left(A y_{\nu}-B x_{\nu}\right) \mathrm{d} \nu-\left(B x_{\mu}-A y_{\mu}\right) \mathrm{d} \mu .
$$

Since closure commutes with pullback, the transformed conservation law is still $\mathrm{d} \Omega_{1}=0$ giving the transformed conservation law

$$
\frac{\partial}{\partial \mu}\left(A y_{\nu}-B x_{\nu}\right)+\frac{\partial}{\partial \nu}\left(B x_{\mu}-A y_{\mu}\right)=0 .
$$


Look at the components of the transformed conservation law

$$
\begin{aligned}
& A y_{\nu}-B x_{\nu}=\frac{1}{2}\left(\phi_{x}^{2}-\phi_{y}^{2}\right) y_{\nu}-\phi_{x} \phi_{y} x_{\nu} \\
& B x_{\mu}-A y_{\mu}=\phi_{x} \phi_{y} x_{\mu}-\frac{1}{2}\left(\phi_{x}^{2}-\phi_{y}^{2}\right) y_{\mu} .
\end{aligned}
$$

Comparison with (7.3) and (7.4) shows that

$$
A y_{\nu}-B x_{\nu}=E_{11} \quad \text { and } B x_{\mu}-A y_{\mu}=E_{12},
$$

verifying that $\delta \mathcal{K} / \delta x=0$ recovers the first of the conservation laws (1.11).

A similar argument verifies that $\delta \mathcal{K} / \delta y=0$ is equivalent to the second conservation law in (1.11) Let $C=\phi_{x} \phi_{y}$ and $D=\frac{1}{2}\left(\phi_{y}^{2}-\phi_{x}^{2}\right)$, then the second conservation law is equivalent to $\mathrm{d} \Omega_{2}=0$ with $\Omega_{2}=C \mathrm{~d} y-D \mathrm{~d} x$. Pullback the $(x, y)$-coordinates to the $(\mu, \nu)$-coordinates,

$$
\Omega_{2}=C\left(y_{\nu} \mathrm{d} \nu+y_{\mu} \mathrm{d} \mu\right)-D\left(x_{\nu} \mathrm{d} \nu+x_{\mu} \mathrm{d} \mu\right)=\left(C y_{\nu}-D x_{\nu}\right) \mathrm{d} \nu-\left(D x_{\mu}-C y_{\mu}\right) \mathrm{d} \mu .
$$

Setting the exterior derivative to zero then gives

$$
\frac{\partial}{\partial \mu}\left(C y_{\nu}-D x_{\nu}\right)+\frac{\partial}{\partial \nu}\left(D x_{\mu}-C y_{\mu}\right)=0 .
$$

The components are

$$
\begin{aligned}
& C y_{\nu}-D x_{\nu}=\phi_{x} \phi_{y} y_{\nu}+\frac{1}{2}\left(\phi_{x}^{2}-\phi_{y}^{2}\right) x_{\nu} \\
& D x_{\mu}-C y_{\mu}=-\frac{1}{2}\left(\phi_{x}^{2}-\phi_{y}^{2}\right) x_{\mu}-\phi_{x} \phi_{y} y_{\mu},
\end{aligned}
$$

which are precisely $E_{21}$ and $E_{22}$.

\section{Boundary variations}

In addition to variations of $\mathscr{L}$ with respect to interior variables and free surface variables there are variations with respect to variables at $\mu=0, \mu=L$ and $\nu=-h$. However they are very similar to variations in physical variables, so just a sketch is given. Consider the case of variations at $\nu=-h$ with the others following similar lines.

Start with the second term in (6.4)

$$
\int_{0}^{L} P_{2}(\mu,-h, \tau) \mathrm{d} \mu=\left.\int_{0}^{L}\left(J g^{12} \phi_{\mu}+J g^{22} \phi_{\nu}\right) \delta \phi\right|_{\nu=-h} \mathrm{~d} \mu .
$$

But $y_{\mu}=0$ since $\nu=-h$ corresponds to $y(\mu,-h, \tau)=-\widehat{h}$, and so

$$
J=x_{\mu} y_{\nu}, \quad g_{11}=x_{\mu}^{2}, \quad g_{12}=x_{\mu} x_{\nu}, \quad \text { at } \quad \nu=-h .
$$

Substituting into the integrand gives

$$
\begin{aligned}
J g^{12} \phi_{\mu}+J g^{22} \phi_{\nu} & =J^{-1}\left(-g_{12} \phi_{\mu}+g_{11} \phi_{\nu}\right) \\
& =\left[-J^{-1} x_{\nu} \phi_{\mu}+J^{-1} x_{\mu} \phi_{\nu}\right] x_{\mu}, \quad \text { at } \quad \nu=-h .
\end{aligned}
$$

Hence

$$
\int_{0}^{L} P_{2}(\mu,-h, \tau) \mathrm{d} \mu=\left.\int_{0}^{L}\left[-J^{-1} x_{\nu} \phi_{\mu}+J^{-1} x_{\mu} \phi_{\nu}\right] \delta \phi\right|_{\nu=-h} x_{\mu} \mathrm{d} \mu,
$$

and so, setting the term in the integrand to zero recovers the bottom boundary condition

$$
-J^{-1} x_{\nu} \phi_{\mu}+J^{-1} x_{\mu} \phi_{\nu}=0 \text { at } \quad \nu=-h .
$$

This is the usual boundary condition of zero normal velocity at a boundary since the left-hand side of $(\mathrm{C}-1)$ is just $\phi_{y}$ in transformed coordinates. 


\section{References}

[1] T.B. Benjamin \& P.J. Olver. Hamiltonian structure, symmetries and conservation laws for water waves, J. Fluid Mech. 125 137-185 (1982).

[2] T.B. Benjamin \& T.J. Bridges. Reappraisal of the Kelvin-Helmholtz problem. Part 1. Hamiltonian structure, J. Fluid Mech. 333 301-325 (1997).

[3] T.J. BRIDGes. Periodic patterns, linear instability, symplectic structure and mean-flow dynamics for $3 D$ surface waves, Phil. Trans. Royal Soc. London A 354 533-574 (1996).

[4] T.J. Bridges. Multi-symplectic structures and wave propagation Math. Proc. Camb. Phil. Soc. 121 147-90 (1997).

[5] T.J. BRIDGes. Conservation laws in curvilinear coordinates: a short proof of Vinokur's Theorem using differential forms, Appl. Math. Comp. 202 882-885 (2008).

[6] T.J. Bridges \& N.M. Donaldson. Mapping techniques in the theory of water waves, Book manuscript (in preparation, 2010).

[7] T.J. Bridges, P.E. Hydon \& J.K. Lawson. Multisymplectic structures and the variational bicomplex, Math. Proc. Camb. Phil. Soc. 148 159-178 (2010).

[8] T.J. Bridges, P.E. Hydon \& S. Reich. Vorticity and symplecticity in Lagrangian fluid dynamics, J. Phys. A: Math. Gen. 38 1403-1418 (2005).

[9] J.G.B. Byatt-Smith. An integral equation for unsteady surface waves and a comment on the Boussinesq equation, J. Fluid Mech. 49 625-633 (1971).

[10] W. Cao, W. Huang \& R.D. Russell. A moving mesh method based on the geometric conservation law, SIAM J. Sci. Comput. 19 118-142 (2002).

[11] W. Cao, W. Huang \& R.D. Russell. Approaches for generating moving adaptive meshes: location versus velocity, Appl. Numer. Math. 47 121-138 (2003).

[12] N.V. Challis \& D.M. Burley. A numerical method for conformal mapping, IMA J. Numer. Anal. 2 169-181 (1982).

[13] W. Choi \& R. Camassa. Exact evolution equations for surface waves, J. Eng. Mech. 125 756-760 (1999)

[14] D. Clamond \& J. Grue. A fast method for fully nonlinear water-wave computations J. Fluid Mech. 447 337-355 (2001).

[15] F. DiAs \& T.J. BRIDGES. The numerical computation of freely propagating time-dependent irrotational water waves, Fluid Dynamics Research 38 803-830 (2006).

[16] A.I. Dyachenko, E.A. Kuznetsov, M.D. Spector \& V.E. Zakharov. Analytical description of the free surface dynamics of an ideal fluid (canonical formalism and conformal mapping), Phys. Lett. A 221 73-79 (1996).

[17] A.I. Dyachenko, V.E. Zakharov \& E.A. Kuznetsov. Nonlinear dynamics of free surface of an ideal fluid, Plasma Physics Reports 22 916-928 (1999).

[18] M. Farrashkhalvat \& J.P. Miles. Basic Structured Grid Generation, ButterworthHeinemann: Oxford (2002). 
[19] G.W. Forbes. On variational problems in parametric form, Amer. J. Phys. 59 1130-1140 (1991).

[20] L.K. Forbes, M.J. Chen \& C.E. Trenham. Computing unstable periodic waves at the interface of two inviscid fluids in uniform vertical flow, J. Comp. Phys. 221 269-287 (2007).

[21] M. Giaquinta \& S. Hildebrandt. Calculus of Variations: the Lagrangian Formalism, Springer-Verlag: Berlin (1996).

[22] M.A. Grant. Standing Stokes waves of maximum height, J. Fluid Mech. 60 593-604 (1973).

[23] M.S. Longuet-Higgins. The instabilities of gravity waves of finite amplitude in deep water. I. Superharmonics, Proc. Roy. Soc. London A 360 471-488 (1978).

[24] M.S. Longuet-Higgins \& E.D. Cokelet. The deformation of steep surface waves on water. I. A numerical method of computation, Proc. Royal Soc. London A 350 1-26 (1976).

[25] J.C. LukE. A variational principle for a fluid with a free surface, J. Fluid Mech. 27 395-397 (1967).

[26] A.V. Marchenko. A Hamiltonian approach to the investigation of the potential motions of an ideal fluid, J. Appl. Maths. Mechs. 59 93-98 (1995).

[27] A. SEIDl \& H. KLOSE. Numerical conformal mapping of a towel-shaped region onto a rectangle, SIAM J. Sci. Stat. Comput. 6 833-842 (1985).

[28] R.V. Shamin. Dynamics of an ideal fluid with a free surface in conformal variables, J. Math. Sci. 160 537-678 (2009).

[29] W. Shyy, H.S. Udaykumar, M.M. Rao \& R.W. Smith. Computational Fluid Dynamics with Moving Boundaries, Dover Publications: New York (1996).

[30] M. TanaKa. The stability of solitary waves, Phys. Fluids 29 650-655 (1986).

[31] P.D. Thomas \& C.K. Lombard. Geometric conservation law and its application to flow computations on moving grids, AIAA Journal 17 1030-1037 (1979).

[32] J.F. Thompson. A survey of dynamically-adaptive grids in the numerical solution of partial differential equations, Appl. Numer. Math. 1 3-27 (1985).

[33] J.F. Thompson, B.K Soni \& N.P. Weatherill. Handbook of Grid Generation, CRC Press: London (1999).

[34] J.-M. Vanden-Broeck. Some new gravity waves in water of finite depth, Phys. Fluids 26 2385-2387 (1983).

[35] V.V. Voinov \& O.V. Voinov. Numerical method of calculating nonstationary motions of an ideal incompressible liquid with free surfaces, Sov. Phys. Dokl. 20 179-180 (1975).

[36] A.K. Whitney. The numerical solution of unsteady free surface flows by conformal mapping, In Proc. Second Inter. Conf. on Numer. Fluid Dynamics (ed. M. HolT), Springer-Verlag 458$462(1971)$.

[37] V.E. Zakharov. Stability of periodic waves of finite-amplitude on the surface of a deep fluid, J. Appl. Mech. Tech. Phys. 9 333-367 (1968). 